\title{
Aquaporin-2 excretion in hospitalized patients with cirrhosis: Relation to development of renal insufficiency and mortality
}

\author{
Troels M Busk, ${ }^{*}{ }^{\dagger}$ Søren Møller, ${ }^{\dagger}$ Erling B Pedersen, ${ }^{\ddagger}$ Alexander Gerbes, ${ }^{\S}$ Aleksander Krag, ${ }^{\natural}$ \\ Markus Peck-Radosavljevic, ${ }^{*}$ Sona Frankova, ${ }^{\dagger \dagger}$ Minneke J Coenraad ${ }^{\text {}}$ and Flemming Bendtsen* \\ ${ }^{*}$ Gastro Unit, Medical Division, ${ }^{\dagger}$ Centre of Functional Imaging and Research, Department of Clinical Physiology and Nuclear Medicine, Copenhagen \\ University Hospital Hvidovre, Hvidovre, ${ }^{\ddagger}$ University Clinic of Nephrology and Hypertension, Aarhus University and Holstebro Hospital, Aarhus, \\ "Department of Gastroenterology and Hepatology, Odense University Hospital, Odense, Denmark; ${ }^{\circledR}$ Liver Center Munich, Klinikum of the University, \\ Ludwig Maximilian University of Munich, Munich, Germany; **Department of Gastroenterology and Hepatology, Endocrinology and Nephrology, \\ Klinikum Klagenfurt am Wörtersee, Klagenfurt, Austria; ${ }^{\dagger}{ }^{\dagger}$ Department of Hepatogastroenterology, Institute for Clinical and Experimental Medicine, \\ Prague, Czech Republic; and ${ }^{\ddagger \neq}$ Department of Gastroenterology and Hepatology, Leiden University Medical Center, Leiden, The Netherlands
}

\author{
Key words \\ aquaporin-2, liver cirrhosis, mortality, renal \\ insufficiency. \\ Accepted for publication 1 November 2016. \\ Correspondence \\ Dr Troels M Busk, Gastro Unit, Medical \\ Division, Copenhagen University Hospital \\ Hvidovre, Kettegaard Alle 30, DK-2650 \\ Hvidovre, Copenhagen, Denmark. \\ Email: troels.malte.busk@regionh.dk; \\ tbusk@hotmail.com
}

Conflicts of interest and financial disclosures: None.

\begin{abstract}
Background and Aim: Urinary aquaporin-2 (AQP2) is a parameter of water transport in the principal cells in the distal part of the nephron and involved in water retention in cirrhosis and may be a marker of renal function. The aim of the study was to evaluate AQP2 as a predictor of renal insufficiency and death in patients with cirrhosis.

Methods: Urine samples from 199 patients (90 patients without organ failure [Group 1], 58 patients with organ failure excluding renal failure [Group 2], and 51 patients with organ failure including renal failure [Group 3]) from the CANONIC study were analyzed for urine $\mathrm{AQP} 2$ and urine osmolality.

Results: There was no difference in AQP2 between the three groups. Urine osmolality was significantly lower in patients in Group 3 versus Group 1 and Group $2(P=0.0004)$. No relation was found between $\mathrm{AQP} 2$ and glomerular filtration rate or creatinine; however, AQP2 was a significant predictor of the development of renal insufficiency $(P=0.0485)$. In a univariate analysis, AQP2 was a significant predictor of 14 and 28-day survival, but this was not confirmed in multivariate analysis.

Conclusions: Aquaporin-2 was not associated with disease severity or markers of renal function but was a predictor for the development of renal insufficiency and death. Therefore, its future use as marker of renal insufficiency could be promising, but further research is needed before it can be considered a clinical useful tool.
\end{abstract}

\section{Introduction}

Renal failure is a frequent complication in liver cirrhosis, often precipitated by infections with sepsis, variceal bleeding, or alcoholic hepatitis. It is a strong marker of poor prognosis. ${ }^{1-3}$

Early diagnosis of renal impairment and identification of the risk of renal failure are important unmet clinical needs.

Serum creatinine is widely used but is an inaccurate marker of glomerular filtration rate (GFR) in cirrhosis. ${ }^{1}$ The limitations of serum creatinine as a marker of renal function in cirrhosis relate to the following: 1) a rise in serum creatinine is delayed following injury; 2) creatinine production is decreased in cirrhotic patients because of malnutrition and muscle wasting; 3 ) the influence of age, gender, and ethnicity, 4) increased renal excretion of serum creatinine in cirrhosis; and 5) determination of serum creatinine interferes with the level of serum bilirubin. ${ }^{1}$

Recently, novel biomarkers in serum or urine, such as neutrophil gelatinase-associated lipocalin ${ }^{4}$ and cystatin $\mathrm{C}^{5}$ reflecting renal function, have been introduced, but prospective studies in larger patient populations with cirrhosis are still missing. Other biomarkers that have been investigated in conditions with fluid retention and renal dysfunction in decompensated cirrhosis include renal water channels such as aquaporin-2 (AQP2).

Aquaporin-2 is located in the collecting ducts in the kidneys and is regulated by vasopressin thereby controlling water permeability across the epithelium. AQP2 is recycled and endocytosed; however, a proportion is excreted in the urine. The excretion is associated with membrane fragments, small vesicles, and multivesicular bodies and not whole-cell shedding in rat urine, but the cellular mechanisms of AQP2 excretion into urine remain unknown. ${ }^{6}$ Dysregulation of AQP2 is seen in several disease entities such as congestive heart failure, syndrome of inappropriate antidiuretic hormone secretion, and liver cirrhosis. ${ }^{7}$ In cirrhosis, AQP2 has been studied in both experimental ${ }^{8-12}$ and human subjects ${ }^{13-20}$ with divergent results with regard to the diagnostic and prognostic value of AQP2.

In this study, we investigated AQP2 as a predictor of renal insufficiency and death, its relation to disease severity, and conventional markers of renal insufficiency in a large cohort of hospitalized cirrhotic patients. 


\section{Patients and methods}

Study population. In the current study, we included 199 patients previously enrolled in the CANONIC study, a prospective, observational study, whose main goal was to establish a definition of acute-on-chronic liver failure (ACLF) in patients with liver cirrhosis admitted with an acute decompensation. ${ }^{21}$ Of the 199 patients, 90 constituted group 1 (G1) that were randomly selected from patients included in the study with an acute decompensation of cirrhosis but without organ failure (group 2 in the CANONIC study). Group 2 (G2) consisted of 58 randomly selected patients with organ failure at inclusion but without renal failure. Patients in this group were originally designated to group 1 in the CANONIC study. Group 3 (G3) consisted of 51 randomly selected patients with organ failure at inclusion including renal failure. $\mathrm{Pa}-$ tients in this group were also originally designated to group 1 in the CANONIC study.

Definitions of organ failure in the CANONIC study were as follows: a) liver failure was defined as a serum bilirubin level of $12 \mathrm{mg} / \mathrm{dL}$ or more; b) kidney failure was defined as a serum creatinine level of $2 \mathrm{mg} / \mathrm{dL}$ or more, or the use of renal replacement therapy; c) cerebral failure was defined as grade III or IV hepatic encephalopathy according to the West Haven criteria; d) coagulation failure was defined as an INR of more than 2.5 and/or platelet count of $20 \times 10^{9} / \mathrm{L}$ or less; e) circulatory failure was defined as the use of dopamine, dobutamine, or terlipressin; f) respiratory failure was defined as a ratio of partial pressure of arterial oxygen to fraction of inspired oxygen $\left(\mathrm{FiO}_{2}\right)$ of 200 or less or a pulse oximetric saturation $\left(\mathrm{SpO}_{2}\right)$ to $\mathrm{FiO}_{2}$ ratio of 200 or less. ${ }^{21}$

In the CANONIC study, ${ }^{21}$ clinical and biochemical data were collected at hospitalization and inclusion (median time between hospitalization and inclusion 3 days) and at different time points during hospitalization (days 1, 2, 3-7, 8-14, 15-21, and 22-28 after inclusion). In this study, we used data from the inclusion visit for analyses. Mortality data from days 7, 14, 28, and 90 were recorded and analyzed. Urine samples were collected 2 days after inclusion into the protocol.

The design of the CANONIC study implies that some patients were admitted because of renal failure, which then was alleviated before inclusion, while other patients developed renal failure in the time between admission and inclusion.

Methods. Glomerular filtration rate was calculated using the following formula:

$$
\begin{aligned}
\text { GFR_MDRD4 }= & 186 \times \text { creatinine }(\mathrm{mg} / \mathrm{dL})^{-1.154} \times \text { age }(\text { years })^{-0.203} \\
& \times \text { GNF } \times \text { ETF },
\end{aligned}
$$

where $\mathrm{GNF}=$ gender factor $($ male $=1$; female $=0.742), \mathrm{ETF}=$ ethnicity factor $($ non-black $=1$; black $=1.21){ }^{22}$

In the analysis of AQP2 as a predictor of renal insufficiency, we retrieved creatinine values at the previously mentioned time points. If any of the patients at these visits had serum creatinine levels above $1.5 \mathrm{mg} / \mathrm{dL}$, they were characterized as having renal insufficiency.

Furthermore, in the analysis of AQP2 as a predictor of renal insufficiency, we applied the recently suggested diagnostic criteria for acute kidney injury (AKI) in patients with cirrhosis.
Definition of AKI is as follows: increase in serum creatinine $\geq 0.3 \mathrm{mg} / \mathrm{dL}(\geq 26.5 \mu \mathrm{mol} / \mathrm{L})$ within $48 \mathrm{~h}$; or a percentage increase in serum creatinine $\geq 50 \%$ from baseline, which is known, or presumed, to have occurred within the prior 7 days. ${ }^{1}$

Lastly, we investigated the impact of the defined organ failures in the Canonic protocol on AQP2.

Urine samples. Urine samples collected on day 2 after inclusion were analyzed for AQP2 and osmolality.

Urine samples were kept frozen at $-20^{\circ} \mathrm{C}$ until assayed; $\mathrm{AQP} 2$ has a half-life of $9-14 \mathrm{~h}^{23}$ and is stable at $-20^{\circ} \mathrm{C}$ for 2 years. ${ }^{6}$ Urinary AQP2 was measured by radioimmunoassay as previously described. ${ }^{24}$ Antibodies were raised in rabbits to a synthetic peptide corresponding to the $15 \mathrm{COOH}$-terminal amino acids in human AQP2 to which was added an NH2-terminal cysteine for conjugation and affinity purification. Minimal detection level was $34 \mathrm{pg} /$ tube. The coefficients of variation were $11.7 \%$ (inter-assay) and $5.9 \%$ (intra-assay). The anti-AQP2 antibody was a gift from Søren Nielsen, Department of Biomedicine, Aarhus University, Denmark.

Plasma and urine osmolality was measured by freezing point depression (Advanced Model 3900 multisampling osmometer).

The CANONIC study was approved by ethical review boards of all study sites, and all patients enrolled in the study gave written consent prior to participation. ${ }^{21}$ The study protocol conforms to the ethical guidelines of the 1975 Declaration of Helsinki as reflected in a priori approval by the institutions human research committees.

Statistics. Data were collected from an electronic case report form.

Results are for the continuous variables given as mean \pm SD if normally distributed and median and interquartile ranges if non-normally distributed. For the categorical variables, results are given as count and percentage in each category. One-way analysis of variance and Kruskal-Wallis test were used for comparison of the continuous variables and Chi-Square test for the categorical variables. Correlations were performed with the Spearman's rank correlation test. For evaluation of AQP2 as a predictor of renal insufficiency and mortality, the MannWhitney test was used for comparison of the two groups. The Mann-Whitney test was also used to evaluate the impact of organ failures on AQP2. Seven-day, 14-day, 28-day, and 90-day mortality rates were estimated as transplant-freecensored mortality using log-rank test to estimate differences between the three groups and Kaplan-Meier plots to demonstrate this graphically. The same approach was applied for the analysis of the differences in AQP2 between the three groups in 28-day and 90-day survival. Univariate analyses of 28-day and 90-day mortality were performed using $t$-test and Mann-Whitney test for continuous variables, normally distributed and non-normally distributed, respectively. For the categorical variables, Chi-Square test or Fisher's exact test was used. Variables resulting statistically significant in the univariate analysis were included in the multivariate analyses, where Cox regression analysis was used. A $P$-value $<0.05$ was considered significant. 


\section{Results}

Table 1 shows the demographic, clinical, and biochemical characteristics of the study population. Of the 199 patients included, 121 were men, and the median age was 57 years (range 48-65); patients in G3 were slightly older than patients in G1 and G2. Patients in G2 and G3 had more advanced disease based upon the MELD score, whereas patients in G2 had higher Child-Pugh score, reflecting the high impact of serum creatinine in the MELD score.

The reason for hospitalization was mainly ascites, followed by hepatic encephalopathy, renal failure, bacterial infections, and gastrointestinal bleeding. In between the groups, there were more patients in G3, who were admitted because of bacterial infections, whereas more patients in G1 were admitted because of gastrointestinal bleeding.

AQP2 and osmolality: distribution and correlation to creatinine and GFR. There was no significant difference in urine AQP2 in the three groups (Fig. 1 and Table 2). Furthermore, when patients were stratified into ACLF or not at inclusion in the study, no difference in AQP2 could be proven: median AQP2 $1.660 \mathrm{ng} / \mathrm{mL}$ (Q1-Q3: 1.120-2.950) versus $1.530 \mathrm{ng} / \mathrm{mL}$ (Q1-Q3: 0.805-3.345), $P=0.3776$. Likewise, there was no difference in AQP2 in patients with or without any of the defined organ failures (renal, coagulation, respiratory, circulatory, or cerebral); only patients with liver failure had borderline significantly higher AQP2 than patients without (median AQP2 $2.260 \mathrm{ng} / \mathrm{mL}$ (Q1$\mathrm{Q} 3: 1.300-4.560)$ versus $1.535 \mathrm{ng} / \mathrm{mL}$ (Q1-Q3: 0.875-3.135), $P=0.0268$.

Urine osmolality was significantly lower in G3 compared with the two other groups $(P=0.0004)$ (Fig. 2 and Table 2).

Aquaporin-2 showed no significant correlation to either creatinine or GFR.

AQP2 and osmolality: relation to mortality. The mortality risk at 7, 14, 28, and 90 days increased progressively throughout the three groups (7-day survival, $P=0.0324$; 14-day survival, $P=0.0004$; 28 -day survival, $P=0.0001$; 90 -day survival, $P=0.0006$, all Tx-censored, $P$-value from log-rank test) with the highest frequencies in G2 and G3 (Fig. 3).

Table 1 Demographic, clinical, and biochemical variables at inclusion

\begin{tabular}{|c|c|c|c|c|c|}
\hline & All Patients $(n=199)$ & $\mathrm{G} 1(n=90)$ & $\mathrm{G} 2(n=58)$ & $\mathrm{G} 3(n=51)$ & $P$-value \\
\hline \multicolumn{6}{|l|}{ Demographic data } \\
\hline Age (years) & $57 \pm 12$ & $56 \pm 12$ & $54 \pm 13$ & $60 \pm 12$ & 0.0409 \\
\hline Sex (male) & $121 / 199(61 \%)$ & $50(56 \%)$ & $40(69 \%)$ & $31(61 \%)$ & 0.2643 \\
\hline \multicolumn{6}{|l|}{ Etiology of cirrhosis } \\
\hline Alcohol & 102/188 (54\%) & $39(47 \%)$ & $37(66 \%)$ & $26(53 \%)$ & 0.0844 \\
\hline $\mathrm{HCV}$ & $36 / 188(19 \%)$ & $20(24 \%)$ & $7(12 \%)$ & $9(18 \%)$ & 0.2310 \\
\hline Alcohol + HCV & 22/188 (12\%) & $11(13 \%)$ & $7(13 \%)$ & $4(8 \%)$ & 0.6631 \\
\hline Miscellaneous & 28/188 (15\%) & $13(16 \%)$ & $5(9 \%)$ & $10(20 \%)$ & 0.2483 \\
\hline \multicolumn{6}{|l|}{ Reason for admission } \\
\hline Ascites & $131 / 197(67 \%)$ & $62(70 \%)$ & $34(60 \%)$ & $35(69 \%)$ & 0.4265 \\
\hline $\mathrm{HE}$ & 68/198 (34\%) & $25(28 \%)$ & $23(40 \%)$ & $20(39 \%)$ & 0.2050 \\
\hline Bacterial infection & $42 / 189(22 \%)$ & $13(15 \%)$ & $12(22 \%)$ & $17(35 \%)$ & 0.0253 \\
\hline GI-bleeding & $32 / 195(16 \%)$ & $21(24 \%)$ & $7(12 \%)$ & $4(8 \%)$ & 0.0270 \\
\hline Renal failure & $58 / 199(29 \%)$ & $11(12 \%)$ & $4(7 \%)$ & $43(84 \%)$ & $<0.0001$ \\
\hline \multicolumn{6}{|l|}{ Clinical and biochemical data } \\
\hline $\mathrm{MAP}(\mathrm{mmHg})$ & $81 \pm 12$ & $81 \pm 11$ & $82 \pm 11$ & $80 \pm 15$ & 0.6738 \\
\hline HR (bpm) & $81 \pm 16$ & $81 \pm 16$ & $82 \pm 14$ & $79 \pm 16$ & 0.6134 \\
\hline Hemoglobin (g/dL) & $10.1 \pm 2.2$ & $10.4 \pm 2.3$ & $9.8 \pm 1.8$ & $10.1 \pm 2.2$ & 0.1952 \\
\hline Platelet count $\left(\times 10^{3} / \mu \mathrm{L}\right)^{\dagger}$ & $78(51-125)$ & $95(58-156)$ & $58(43-104)$ & $79(61-143)$ & 0.0007 \\
\hline $\operatorname{ALT}(U / L)^{\dagger}$ & $32(21-50)$ & $32(20-45)$ & $35(24-51)$ & $30(19-49)$ & 0.6108 \\
\hline Bilirubin $(\mathrm{mg} / \mathrm{dL})^{\dagger}$ & $3.0(1.6-8.2)$ & $2.0(1.4-4.7)$ & $7.2(3.4-14.4)$ & $2.6(1.4-8.3)$ & $<0.0001$ \\
\hline Serum albumin (g/dL) & $2.9 \pm 0.6$ & $2.8 \pm 0.6$ & $2.8 \pm 0.6$ & $3.0 \pm 0.7$ & 0.0700 \\
\hline $\mathrm{INR}^{+}$ & $1.6(1.3-2.1)$ & $1.8(1.4-2.5)$ & $2.7(2.3-4.7)$ & $2.0(1.5-4.0)$ & $<0.0001$ \\
\hline Alkaline phosphatase $(U / L)^{\dagger}$ & $146(89-213)$ & $146(102-239)$ & $156(88-235)$ & $136(88-190)$ & 0.4935 \\
\hline \multicolumn{6}{|l|}{ Liver scores } \\
\hline MELD score & $21 \pm 8$ & $15 \pm 5$ & $24 \pm 7$ & $27 \pm 8$ & $<0.0001$ \\
\hline Child-Pugh score & $10 \pm 2$ & $9 \pm 2$ & $11 \pm 2$ & $10 \pm 2$ & $<0.0001$ \\
\hline
\end{tabular}

Results are given as mean $\pm \mathrm{SD}$ or median $(\mathrm{Q} 1-\mathrm{Q} 3)$ if not normally distributed variable $={ }^{\dagger}$. P-value from ANOVA or Kruskal-Wallis test if not normally distributed variable $=^{\dagger}$.

For categorical variables: $n(\%)$. P-value from Chi-Square test.

G1: patients without organ failure at inclusion; G2: patients with organ failure excluding renal failure at inclusion; G3: patients with organ failure including renal failure at inclusion.

ALT, alanine transaminase; GI-bleeding, gastrointestinal bleeding; HCV, hepatitis C virus; HE, hepatic encephalopathy; HR, heart rate; MAP, mean arterial pressure; MELD, model for end-stage liver disease. 


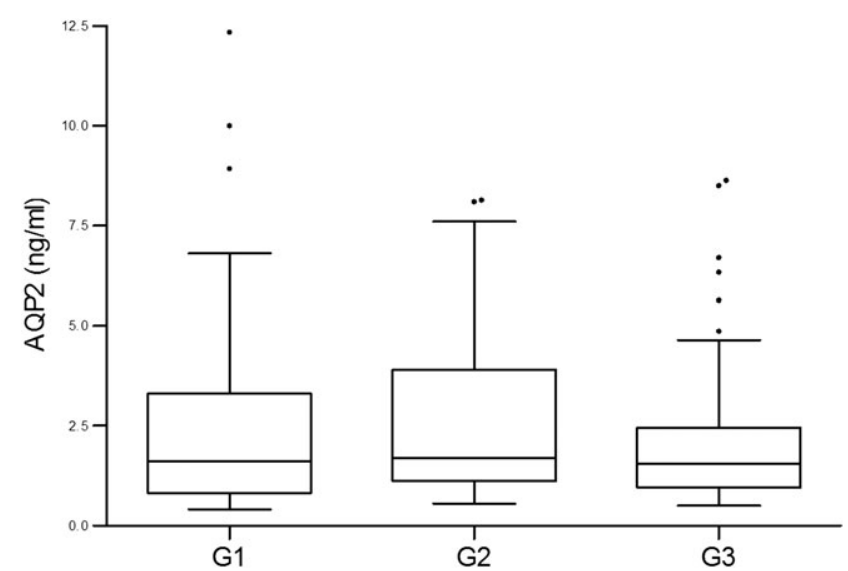

Figure 1 Urine AOP2 levels in G1 (patients without organ failure at inclusion), G2 (patients with organ failure excluding renal failure at inclusion), and G3 (patients with organ failure including renal failure at inclusion). Squares are outliers.

In the univariate analysis, AQP2 predicted 14 and 28-day mortality significantly $(P=0.0420$ and 0.0322 , respectively) where an increase in AQP2 was related to increased mortality; see Table 3. However, this was not confirmed in a multivariate analysis. Other factors statistically significant in univariate analysis of both 28 and 90-day mortality were reason for admission: renal failure, hemoglobin, bilirubin, INR, serum creatinine, GFR, MELD score, and Child-Pugh score; see Table 3.

Urine osmolality showed statistical significance as a predictor of 90-day mortality in univariate analysis $(P=0.004)$ and remained significant in a model together with hemoglobin, creatinine, and Child-Pugh score in the multivariate analysis; see Tables 3 and 4.

AQP2 as a predictor of renal insufficiency. Of the 199 patients included, 130 patients had serum creatinine levels below $1.5 \mathrm{mg} / \mathrm{dL}$ at inclusion. Of these 130 patients, 22 developed renal insufficiency, defined as creatinine above $1.5 \mathrm{mg} / \mathrm{dL}$ at some point of time during follow-up. However, baseline AQP2 was unable to discriminate patients, who developed renal insufficiency from patients not developing renal insufficiency: median AQP2 $2.430 \mathrm{ng} / \mathrm{mL}$ (Q1-Q3: 1.460-3.710) versus $1.385 \mathrm{ng} / \mathrm{mL}$ (Q1-Q3: $0.860-3.820), P=0.2122$.

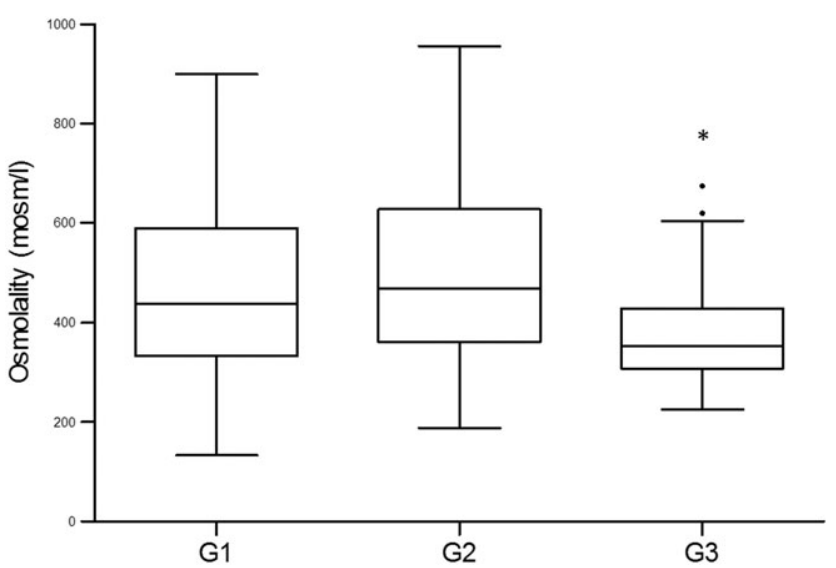

Figure 2 Urine osmolality levels in G1 (patients without organ failure at inclusion), G2 (patients with organ failure excluding renal failure at inclusion), and G3 (patients with organ failure including renal failure at inclusion). Squares are outliers. ${ }^{*} P=0.0004, \mathrm{G} 3$ versus $\mathrm{G} 1$ and $\mathrm{G} 2$.

When we applied the recently suggested diagnostic criteria for AKI in patients with cirrhosis, 58 patients developed AKI at some point of time during follow-up. In this analysis, baseline AQP2 was able to discriminate patients, who developed AKI from patients who did not: median AQP2 $1.870 \mathrm{ng} / \mathrm{mL}$ (Q1Q3: 1.200-3.550) versus $1.425 \mathrm{ng} / \mathrm{mL}$ (Q1-Q3: 0.805-3.250), $P=0.0485$.

\section{Discussion}

The main findings of the study were that, in cirrhosis, 1) AQP2 was not related to disease severity, that is there was no difference in the urinary concentration of AQP2 between the three groups or between patients with or without ACLF; 2) AQP2 was able to predict development of renal insufficiency according to recently proposed diagnostic criteria; 3) although $\mathrm{AQP} 2$ in a univariate analysis could predict 14 and 28-day mortality, this did not hold true in a multivariate analysis; and 4) AQP2 did not correlate with conventional markers of renal function, that is GFR and serum creatinine.

The diagnostic criteria of the hepatorenal syndrome (HRS) and AKI have been debated and revised several times over the years primarily to improve early identification and correct classification of renal failure in order to apply focused therapy. ${ }^{1,25-27}$ Serum

Table 2 Urine AQP2, urine osmolality, and renal parameters at inclusion

\begin{tabular}{|c|c|c|c|c|c|}
\hline & All patients ( $n=199$ ) & $\mathrm{G} 1(n=90)$ & $\mathrm{G} 2(n=58)$ & $\mathrm{G} 3(n=51)$ & $P$-value \\
\hline Urine AQP2 $(\mathrm{ng} / \mathrm{mL})^{\dagger}$ & $1.58(0.92-3.27)$ & $1.62(0.82-3.27)$ & $1.71(1.12-3.79)$ & $1.55(0.96-2.45)$ & 0.4938 \\
\hline Urine osmolality (mosm/L) & $457 \pm 168$ & $465 \pm 185$ & $507 \pm 161$ & $382 \pm 110$ & 0.0004 \\
\hline Serum creatinine $(\mathrm{mg} / \mathrm{dL})^{\dagger}$ & $1.0(0.7-2.0)$ & $0.9(0.7-1.1)$ & $0.9(0.7-1.1)$ & $2.9(2.4-3.7)$ & $<0.0001$ \\
\hline GFR $\left(\mathrm{mL} / \mathrm{min} / 1.73 \mathrm{~m}^{2}\right)^{\dagger}$ & $71(32-105)$ & $90(66-112)$ & $90(66-115)$ & $20(16-27)$ & $<0.0001$ \\
\hline
\end{tabular}

Results are given as mean \pm SD or median (Q1 - Q3) if not normally distributed variable $={ }^{\dagger}$. P-value from ANOVA or Kruskal-Wallis test if not normally distributed variable $=^{\dagger}$.

G1: patients without organ failure at inclusion; G2: patients with organ failure excluding renal failure at inclusion; G3: patients with organ failure including renal failure at inclusion.

GFR, glomerular filtration rate. 


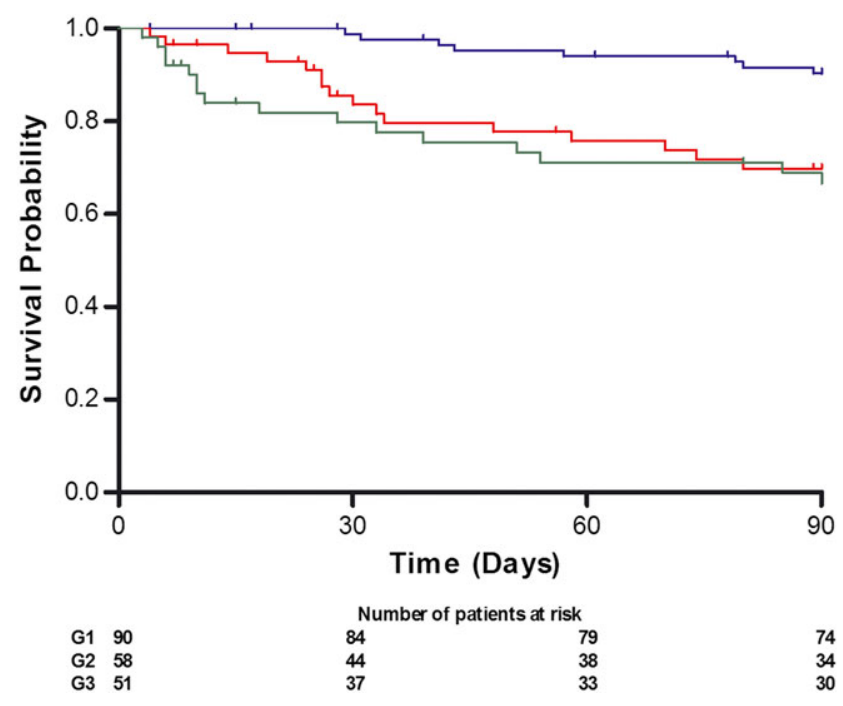

Figure 3 Ninety-day survival (Tx-censored) by groups of analyses. $P$-value (log-rank test) $=0.0006 . \_, G 1$ (patients without organ failure at inclusion), —, G2 (patients with organ failure excluding renal failure at inclusion), and - - G3 (patients with organ failure including renal failure at inclusion). [Color figure can be viewed at wileyonlinelibrary.com]

creatinine is still widespread used for clinical assessment of renal function, although it is a poor marker of especially moderate renal insufficiency in decompensated cirrhotic patients, where it tends to overestimate GFR. Moreover, the alternatives, for example creatinine clearance based on 24-h urine collection, and more advanced techniques like ${ }^{51} \mathrm{Cr}$-EDTA clearance are associated with a number of disadvantages. Increased tubular creatinine secretion in cirrhosis makes estimation of creatinine clearance unreliable, and moreover, the urine collection is often incomplete. ${ }^{51} \mathrm{Cr}$-EDTA clearance is the gold standard of GFR assessment, but because of complexity in analysis unsuitable for many repeated measurements. ${ }^{2,27}$

Aquaporin-2 plays a central role in the regulation of free-water clearance. Its pathophysiological role has been studied in many diseases, amongst these in cirrhosis with contrasting results.
Table 4 Ninety-day survival, multivariate analysis

\begin{tabular}{lcll}
\hline Tested variables & $N$ (events) & HR & $(95 \%$ IC) \\
\hline Osmolality & 162 (31) & 0.997 & $0.995-1.000$ \\
Hemoglobin & - & 0.852 & $0.726-0.999$ \\
Log serum creatinine $^{\dagger}$ & - & 2.203 & $1.269-3.824$ \\
Child-Pugh score $^{+}$ & - & 1.558 & $1.280-1.895$ \\
\hline
\end{tabular}

${ }^{\dagger}$ Serum creatinine was log-transformed (Log) in order to follow a normal distribution.

In animal studies, Fujita et al. and Asahina et al. ${ }^{8,11}$ found increased levels of AQP2 protein and AQP2 mRNA in rats with $\mathrm{CCl}_{4}$-induced cirrhosis, whereas Jonassen et al. and FernandezLlama et al. in rats with bile duct ligated-induced cirrhosis showed decreased expression of AQP2. ${ }^{9,12}$ The latter group could not in a later study with rats with $\mathrm{CCl}_{4}$-induced cirrhosis demonstrate any difference in the abundance of AQP2; however, seemingly, there was a redistribution of AQP2 indicative of AQP2 trafficking to the apical plasma membrane, resulting in water retention. ${ }^{10}$ The differences found in these animal studies may partly be due to the ways by which cirrhosis is induced (bile duct ligated vs $\mathrm{CCl}_{4}$ ).

Ivarsen et al. and Pedersen et al. were the first to describe changes in AQP2 in cirrhotic patients. ${ }^{15,16}$ Ivarsen et al. found an increased excretion of AQP2 in urine in 33 cirrhotic patients with a progressive increase throughout the Child classes. Patients were on their usual medication including diuretics as in the present study. It still needs to be clarified, whether urinary AQP2 excretion is affected by the intake of diuretics, because one study showed no effect, ${ }^{28}$ and others have found the opposite. ${ }^{29}$ The differences in findings between the study by Ivarsen et al. and ours may be due to our patients being divided into groups based on various degrees of organ failures and not the Child-Pugh score.

Pedersen et al. did not find any difference in AQP2 excretion between 14 stable cirrhotic patients compared with healthy adults; in their study, medication was withdrawn $24 \mathrm{~h}$ prior to investigations. $^{16}$

Esteva-Font et al. ${ }^{13}$ in 20 cirrhotic patients observed a lower excretion of AQP2 in urine compared with healthy subjects with a

Table 3 Twenty-eight-day and 90-day survival, univariate analysis

\begin{tabular}{|c|c|c|c|c|c|c|}
\hline & \multicolumn{3}{|c|}{ 28-day mortality } & \multicolumn{3}{|c|}{ 90-day mortality } \\
\hline & Alive $(n=181)$ & Dead $(n=18)$ & $P$-value & Alive $(n=156)$ & Dead $(n=40)$ & $P$-value \\
\hline Urine AQP2 $(\mathrm{ng} / \mathrm{mL})^{+}$ & $1.50(0.89-3.24)$ & $2.32(1.55-4.2)$ & 0.0322 & $1.55(0.89-3.24)$ & $1.77(1.17-3.47)$ & 0.3747 \\
\hline Urine osmolality (mosm/L) & $462 \pm 171$ & $404 \pm 119$ & 0.1764 & $469 \pm 178$ & $402 \pm 107$ & 0.004 \\
\hline Reason for admission: renal failure & $48 / 181(26 \%)$ & $10 / 18(56 \%)$ & 0.0097 & $39 / 156(25 \%)$ & $18 / 40(45 \%)$ & 0.0130 \\
\hline Hemoglobin $(\mathrm{g} / \mathrm{dL})$ & $10.3 \pm 2.1$ & $8.9 \pm 2.3$ & 0.0105 & $10.4 \pm 2.2$ & $9.2 \pm 1.9$ & 0.001 \\
\hline Bilirubin $(\mathrm{mg} / \mathrm{dL})^{\dagger}$ & $2.7(1.5-7.3)$ & $10.6(3.1-24)$ & 0.0010 & $2.4(1.5-7.1)$ & $6.8(2.6-16.5)$ & $<0.0001$ \\
\hline $\mathrm{INR}^{+}$ & $1.5(1.3-2.0)$ & $2.1(1.5-2.6)$ & 0.0029 & $1.5(1.3-1.8)$ & $2.1(1.5-2.6)$ & $<0.0001$ \\
\hline Serum creatinine $^{\dagger}(\mathrm{mg} / \mathrm{dL})$ & $0.9(0.7-1.9)$ & $2.4(1.1-3.7)$ & 0.0002 & $1.0(0.7-1.8)$ & $1.8(0.9-3.0)$ & 0.0040 \\
\hline $\operatorname{GFR}\left(\mathrm{mL} / \mathrm{min} / 1.73 \mathrm{~m}^{2}\right)^{\dagger}$ & $81(37-109)$ & $29(19-63)$ & 0.0001 & $82(38-110)$ & $37(20-85)$ & 0.0022 \\
\hline MELD Score & $20 \pm 7$ & $30 \pm 8$ & $<0.0001$ & $19 \pm 7$ & $27 \pm 8$ & $<0.0001$ \\
\hline Child-Pugh score & $10 \pm 2$ & $12 \pm 1$ & 0.0017 & $10 \pm 2$ & $11 \pm 2$ & $<0.0001$ \\
\hline
\end{tabular}

Results are given as mean $\pm \mathrm{SD}$ or median $(\mathrm{Q} 1-\mathrm{Q} 3)$ if not normally distributed variable $={ }^{\dagger}$. $P$-value from T-test or U-Mann-Whitney test if not normally distributed variable $=^{\dagger}$.

For categorical variables: $n(\%)$. P-value from Chi-Square test.

GFR, glomerular filtration rate; MELD, model for end-stage liver disease. 
progressive decrease in the excretion of AQP2 with increasing disease severity; patients without ascites had the highest levels of excreted AQP2, followed by patients with ascites and patients with HRS. In this study, all medication including diuretics was withdrawn 5 days prior to investigation, while patients were allowed to drink freely. This standardized condition is however not feasible in practical daily care.

Chung et al., ${ }^{17}$ in coherence with Ivarsen et al., found higher levels of AQP2 in urine in 81 cirrhotic patients compared with healthy subjects, and patients with ascites had higher levels compared with patients without ascites. There was no difference in AQP2 between patients who were kept on medication including diuretics compared with those where medication was withdrawn prior to the study.

In our study, AQP2 was not related to disease severity, neither when based upon our three patient groups, that is patients without organ failure, with organ failure excluding renal failure, and with organ failure including renal failure, nor when patients were divided according to having ACLF or not. Furthermore, AQP2 was unable to predict development of death, and it was not related to conventional markers of renal function. However, AQP2 was able to predict development of renal insufficiency, although only borderline significant.

The obvious strength of our study is the prospective design of the study and the large number of patients included.

Furthermore, patients were not studied in standardized conditions with regards to other conditions, for example hydration status and cessation of diuretics. This is important, because a biomarker of renal function should be useful in a clinical setting.

There are many potential reasons for the negative findings in our study. Dehydration or water deprivation is known to decrease urinary AQP2 excretion. ${ }^{13,15}$ In our study, the hydration level of the patients was unknown, and possibly, some patients were dehydrated. Urinary AQP2 excretion may reflect abundance in renal collecting ducts but not necessarily activity.

Medication that could have influenced AQP2 was not withdrawn, and most likely, many of the patients in G3 with probable HRS and possibly those admitted with gastrointestinal bleeding would have received treatment with vasopressin analogues and human albumin, known to affect the excretion of AQP2 into urine, although minor. ${ }^{18}$ Moreover, diuretics were not withdrawn, which could also have influenced the results, although the effect on urinary AQP2 excretion has been questioned. $^{28,29}$

Furthermore, urine samples were collected up to 2 days after inclusion in the study. As ACLF is a very dynamic syndrome, ${ }^{30}$ these "delays" could have influenced the AQP2 level and thus blurred the results.

We did not correct for the creatinine concentration in urine. This would have enabled us to correct for the variation in the volume of the diuresis that naturally occurs during a 24-h period, which affects the excretion of AQP2 in urine. Another way to come about this matter is to use the excretion of AQP2 in urine over a certain time period, which was not possible.

In a clinical perspective, our findings could support the use of AQP2 as a marker of renal insufficiency in clinical practice, although further research hereof is needed. This would moreover improve our understanding of the handling of water and sodium excretion in cirrhosis. ${ }^{18-20}$
The finding of low urine osmolality to be associated with renal insufficiency and thereby death is not surprising, because decompensated patients are characterized by their inability to concentrate their urine. Thus, low urine osmolality is probably a phenomenon in the established pathophysiology of severe decompensated cirrhosis with renal failure. ${ }^{13}$

In conclusion, AQP2 does predict development of renal insufficiency but does not correlate to conventional markers of renal insufficiency. Therefore, its future use as a marker of renal function is unclear, but potentially promising, perhaps in combination with other markers of renal function such as Cystatin $\mathrm{C}$ and neutrophil gelatinase-associated lipocalin.

\section{Acknowledgments}

Søren Møller was supported by a grant from the Novo Nordisk Foundation.

\section{Reference}

1 Angeli P, Gines P, Wong F et al. Diagnosis and management of acute kidney injury in patients with cirrhosis: revised consensus recommendations of the International Club of Ascites. Gut 2015; 64: 531-7.

2 Gines P, Schrier RW. Renal failure in cirrhosis. N. Engl. J. Med. 2009; 361: 1279-90.

3 Fede G, D'Amico G, Arvaniti V et al. Renal failure and cirrhosis: a systematic review of mortality and prognosis. J. Hepatol. 2012; 56 : 810-18.

4 Gerbes AL, Benesic A, Vogeser M, Krag A, Bendtsen F, Moller S. Serum neutrophil gelatinase-associated lipocalin - a sensitive novel marker of renal impairment in liver cirrhosis? Digestion 2011; 84: 82-3.

5 Randers E, Ivarsen P, Erlandsen EJ et al. Plasma cystatin C as a marker of renal function in patients with liver cirrhosis. Scand. J. Clin. Lab. Invest. 2002; 62: 129-34.

6 Graffe CC, Bech JN, Pedersen EB. Effect of high and low sodium intake on urinary aquaporin-2 excretion in healthy humans. Am. $J$. Physiol. Renal. Physiol. 2012; 302: F264-F275.

7 Knepper MA, Kwon TH, Nielsen S. Molecular physiology of water balance. N. Engl. J. Med. 2015; 372: 1349-58.

8 Asahina Y, Izumi N, Enomoto N et al. Increased gene expression of water channel in cirrhotic rat kidneys. Hepatology 1995; 21: 169-73.

9 Fernandez-Llama P, Turner R, Dibona G, Knepper MA. Renal expression of aquaporins in liver cirrhosis induced by chronic common bile duct ligation in rats. J. Am. Soc. Nephrol. 1999; 10: 1950-7.

10 Fernandez-Llama P, Jimenez W, Bosch-Marce M, Arroyo V, Nielsen $\mathrm{S}$, Knepper MA. Dysregulation of renal aquaporins and $\mathrm{Na}-\mathrm{Cl}$ cotransporter in CCl4-induced cirrhosis. Kidney Int. 2000; 58: 216-28.

11 Fujita N, Ishikawa SE, Sasaki S et al. Role of water channel AQP-CD in water retention in SIADH and cirrhotic rats. Am. J. Physiol. 1995; 269: F926-F931.

12 Jonassen TE, Brond L, Torp M et al. Effects of renal denervation on tubular sodium handling in rats with CBL-induced liver cirrhosis. Am. J. Physiol. Renal. Physiol. 2003; 284: F555-F563.

13 Esteva-Font C, Baccaro ME, Fernandez-Llama P et al. Aquaporin-1 and aquaporin-2 urinary excretion in cirrhosis: Relationship with ascites and hepatorenal syndrome. Hepatology 2006; 44: 1555-63.

14 Geilswijk M, Thomsen KL, Pedersen EB, Vilstrup H, Gronbaek H. Urinary aquaporin-2 excretion before and after transjugular intrahepatic portosystemic shunt insertion for refractory ascites. Scand. J. Gastroenterol. 2015; 50: 454-61. 
15 Ivarsen P, Frokiaer J, Aagaard NK et al. Increased urinary excretion of aquaporin 2 in patients with liver cirrhosis. Gut 2003; 52: 1194-9.

16 Pedersen RS, Bentzen H, Bech JN, Nyvad O, Pedersen EB. Urinary aquaporin-2 in healthy humans and patients with liver cirrhosis and chronic heart failure during baseline conditions and after acute water load. Kidney Int. 2003; 63: 1417-25.

17 Chung SH, Jun DW, Kim KT et al. Aquaporin-2 urinary excretion in cirrhosis: relationship to vasopressin and nitric oxide. Dig. Dis. Sci. 2010; 55: 1135-41.

18 Krag A, Bendtsen F, Pedersen EB, Holstein-Rathlou NH, Moller S. Effects of terlipressin on the aquaretic system: evidence of antidiuretic effects. Am. J. Physiol. Renal. Physiol. 2008; 295: F1295-F1300.

19 Krag A, Moller S, Pedersen EB, Henriksen JH, Holstein-Rathlou NH, Bendtsen F. Impaired free water excretion in child $\mathrm{C}$ cirrhosis and ascites: relations to distal tubular function and the vasopressin system. Liver Int. 2010; 30: 1364-70.

20 Krag A, Pedersen EB, Moller S, Bendtsen F. Effects of the vasopressin agonist terlipressin on plasma cAMP and ENaC excretion in the urine in patients with cirrhosis and water retention. Scand. J. Clin. Lab. Invest. 2011; 71: 112-16.

21 Moreau R, Jalan R, Gines P et al. Acute-on-chronic liver failure is a distinct syndrome that develops in patients with acute decompensation of cirrhosis. Gastroenterology 2013; 144: 1426-37.1437

22 Levey AS, Coresh J, Greene T et al. Using standardized serum creatinine values in the modification of diet in renal disease study equation for estimating glomerular filtration rate. Ann. Intern. Med. 2006; 145: 247-54.
23 Sandoval PC, Slentz DH, Pisitkun T, Saeed F, Hoffert JD, Knepper MA. Proteome-wide measurement of protein half-lives and translation rates in vasopressin-sensitive collecting duct cells. J. Am. Soc. Nephrol. 2013; 24: 1793-805.

24 Pedersen RS, Bentzen H, Bech JN, Pedersen EB. Effect of water deprivation and hypertonic saline infusion on urinary AQP2 excretion in healthy humans. Am. J. Physiol. Renal. Physiol. 2001; 280: F860-F867.

25 Arroyo V, Gines P, Gerbes AL et al. Definition and diagnostic criteria of refractory ascites and hepatorenal syndrome in cirrhosis. Int Ascites Club Hepatol. 1996; 23: 164-76.

26 Salerno F, Gerbes A, Gines P, Wong F, Arroyo V. Diagnosis, prevention and treatment of hepatorenal syndrome in cirrhosis. Gut 2007; 56: 1310-8.

27 Wong F, Nadim MK, Kellum JA et al. Working Party proposal for a revised classification system of renal dysfunction in patients with cirrhosis. Gut 2011; 60: 702-9.

28 Marples D, Christensen BM, Frokiaer J, Knepper MA, Nielsen S. Dehydration reverses vasopressin antagonist-induced diuresis and aquaporin-2 downregulation in rats. Am. J. Physiol. 1998; 275: F400-F409.

29 Starklint J, Bech JN, Pedersen EB. Urinary excretion of aquaporin-2 after furosemide and felodipine in healthy humans. Scand. J. Clin. Lab. Invest. 2005; 65: 249-61.

30 Gustot T, Fernandez J, Garcia E et al. Clinical Course of acute-onchronic liver failure syndrome and effects on prognosis. Hepatology 2015; 62: 243-52. 